\title{
Use of Nelder-Mead simplex method to arc fitting for railway track realignment
}

\author{
Marta Skała-Szymańska, Sławomir Cellmer, Jacek Rapiński \\ University of Warmia and Mazury, ul. Oczapowskiego 1/25, Olsztyn 10-719, Poland
}

\begin{abstract}
The development of technology implies the elaboration of new calculation methods. Such methods should be precise and efficient. The article presents a new method of railway track realignment. The example considered in the article consistsof an circular arc that is tangent to two straight lines. Authors show subsequent stages of realization of tasks of railway track realignment. Each formula is described in this paper. The novelty of this solution is use of the Nelder-Mead simplex method for fitting section of railway track geometry into set of measure points. The functional model of optimization problem was derived and discussed in details. The method of optimization problem was presented in the form of detailed algorithm. The example of use of proposed algorithm was presented and analysed.
\end{abstract}

Keywords: optimize; Nelder-Mead simplex method; railway track realignment.

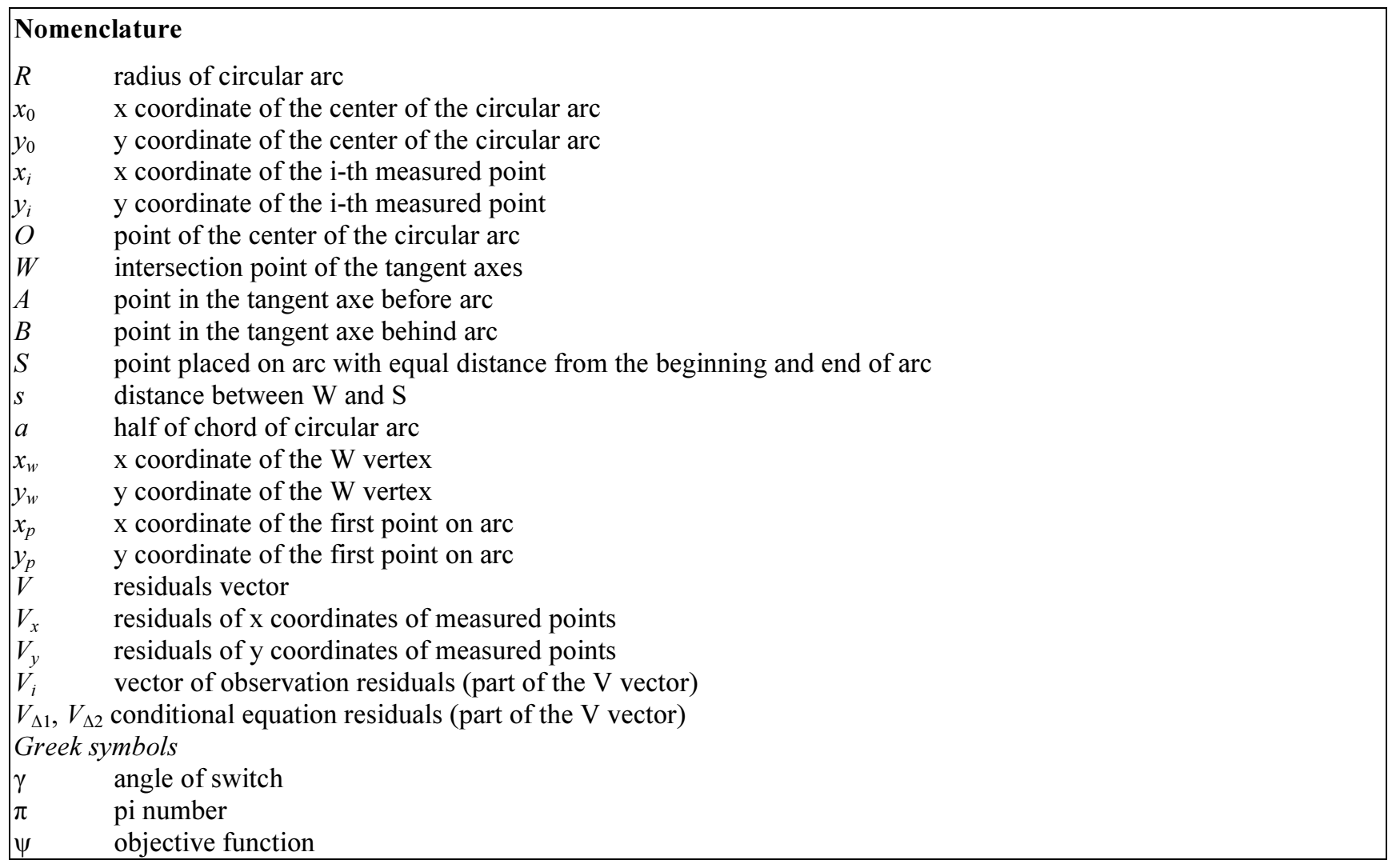

Corresponding author: Marta Skała-Szymańska. E-mail address: marta.skala@uwm.edu.pl http://dx.doi.org/10.3846/enviro.2014.244

(C) 2014 The Authors. Published by VGTU Press. This is an open-access article distributed under the terms of the Creative Commons Attribution License, which permits unrestricted use, distribution, and reproduction in any medium, provided the original author and source are credited. 


\section{Introduction}

Nowadays, a number of railway tracks modernization is carried out. New railway lines are also being built. The article presents a new approach to fitting of a circular arc into a set of measured points. So far, the methods used for realignment of railway tracks have applied time-consuming techniques, i.e. graphical method, semi-numerical method and mechanical method. The most commonly used method is semi-numerical. In this case the diagrams of angles are drawn. They are designed on the basis of some curve geometry parameters which are measured in terrain [7]. The main disadvantage of this method is it's randomness (high risk of obtaining a solution which is not optimal) and low accuracy when applied to the case of nonlinear curves [5]. Another methods are methods based on graph: curvatures, sagittas and track cants, but these type of graphs shows a high sensitivity to the calculation and measurement methods. Lenda and Strach in [3] presented a method based on graph of curvature created by approximate of a spline function of a railway track. This allows to avoid the ambiguity associated with the assignment of appropriate curvature to the points. The desirable method should fulfill a number of assumptions, especially those that eliminate manual measurement of sagittas. Existing methods do not allow for such an approach [8]. Currently used methods are low efficient. This is due to e.g. the nature of the nonlinear function for fitting a set of curves. This problem is eliminated by linearization of such functions, which causes a loss of accuracy [6]. Another possible approach is to use optimization method for nonlinear functions. Unfortunately such methods require a lot of calculations. In the article the new method is proposed, which use nonlinear functions and an efficient calculation algorithm. Due to the development of satellite technology Specht et al. developed a new method of the railroad tracks realignment using continuous satellite measurements with antennas installed on a moving rail vehicle. This allows to restore the direct axis position of tracks in an absolute reference frame after eliminating the Cross Track Error (XTE) [1]. A new element in this proposal is use the Nelder-Mead simplex method (often called the ameba simplex method) for searching for the optimal solution. This method was described in the following chapter.

\section{The Nelder-Mead Simplex Method}

The novelty of this research lies in a different approach to the task of railroad tracks realignment. Methods were used so far based on the use of graphic methods. In contrast to other methods based on objective function linearization, which results in a loss of accuracy the Nelder-Mead simplex method is numerical and direct. The algorithm does not use derivatives, so in a simple manner it works with nonlinear functions. It is based only on calculating of the values of the objective function [2]. Finally, in a structured way tends to a global minimum. The Nelder-Mead simplex method starts with forming a simplex (set of points) in a multi-dimensional space. The number of vertices of the simplex is by one bigger than the dimension of the parameter space. The number of parameters is equal to the dimension of space. For example: one-dimensional simplex has two vertices and this is the section of a straight line, two-dimensional simplex has three vertices, so it is a triangle. Generally, the $n$ dimensional simplex of the $n+1$ vertices is a polyhedron represented by $n+1$ vectors.

At the beginning the initial simplex is formed, assuming a certain distance between the vertices (the default is five percent of the parameter value). Then the transformations of the simplex are carried out as long as the distance between the vertices is less than the assumed accuracy of the calculations. During optimization procedure simplex is modified many times, until it reaches the global minimum. In procedure of searching for the minimum of objective function the following operations are applied: calculating the center of gravity of the simplex, reflection, expansion, contraction and reduction. In order to determine the minimum in the first iteration must be performed in sequence [4]:

- organizing the function values at the vertices and calculating the center of gravity of the simplex $p_{0}$ without a $p_{\text {high }}(1$ with Fig. 1),

$$
f\left(p_{\text {low }}\right) \leq \ldots \leq f\left(p_{i}\right) \leq f\left(p_{\text {high }}\right)
$$

where:

$p_{\text {low }}=p_{1} ; p_{\text {low }}$ is a point of simplex, where the function has a minimum value,

$p_{\text {high }}=p_{n+1} ; p_{\text {high }}$ is a point of simplex, where the function has a maximum value.

- reflection - searching a new point $p_{\mathrm{r}}(2$ with Fig. 1),

$$
p_{r}=p_{0}+\alpha\left(p_{0}-p_{h i g h}\right)
$$

where:

$$
\alpha=1 \text { and it is a reflection coefficient. }
$$

If $f\left(p_{i}\right)>f\left(p_{r}\right) \geq f\left(p_{\text {low }}\right)$, then a new simplex is determined using $\mathrm{p}_{\mathrm{r}}$ in place of $p_{\text {high }}$ and go to step 1 . But if $f\left(p_{r}\right)<f\left(p_{\text {low }}\right)$, then go to step 3 .

- expansion - calculating a $p_{e}$ (3 with Fig. 1),

$$
p_{e}=p_{0}+\beta\left(p_{0}-p_{\text {high }}\right)
$$


where:

$$
\beta=2 \text { and it is a expansion coefficient. }
$$

If $f\left(p_{e}\right)<f\left(p_{r}\right)$ then determine a new simplex with $p_{\mathrm{e}}$ instead of $p_{\text {high, }}$ then go back to step 1 . In another case, determine the simplex containing $p_{r}$ in place $p_{\text {high }}$ and go back to step 1 .

- contraction (4 with Fig. 1),

if $f\left(p_{r}\right) \geq f\left(p_{i}\right)$ and $p_{\mathrm{i}} \neq p_{\text {high }}$, then computing a $p_{c}$ :

$$
p_{c}=p_{h i g h}+\rho\left(p_{0}-p_{h i g h}\right)
$$

where:

$\rho=0.5$ and it is a contraction coefficient.

But if $f\left(p_{c}\right) \leq f\left(p_{\text {high }}\right)$ then determine a new simplex containing $\mathrm{p}_{\mathrm{c}}$ in place of $p_{\text {high }}$, then go back to step 1. Otherwise, go to step 5 .

- reduction - forming a new, smaller simplex whose vertices are $p_{1(l o w)}, p_{2(N E W),} p_{3(N E W)}(5$ with Fig. 1),

$$
p_{i}=p_{\text {low }}+\sigma\left(p_{i}-p_{\text {low }}\right)
$$

where:

$\sigma=0.5$ and it is a reduction coefficient,

$i \in\{2, \ldots, n+1\}$.

ese operations (1-5) are shown graphically on figure below:

1) initial simplex

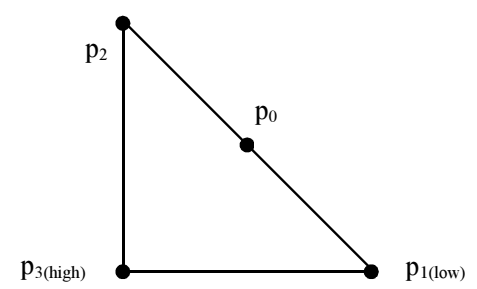

3) expansion

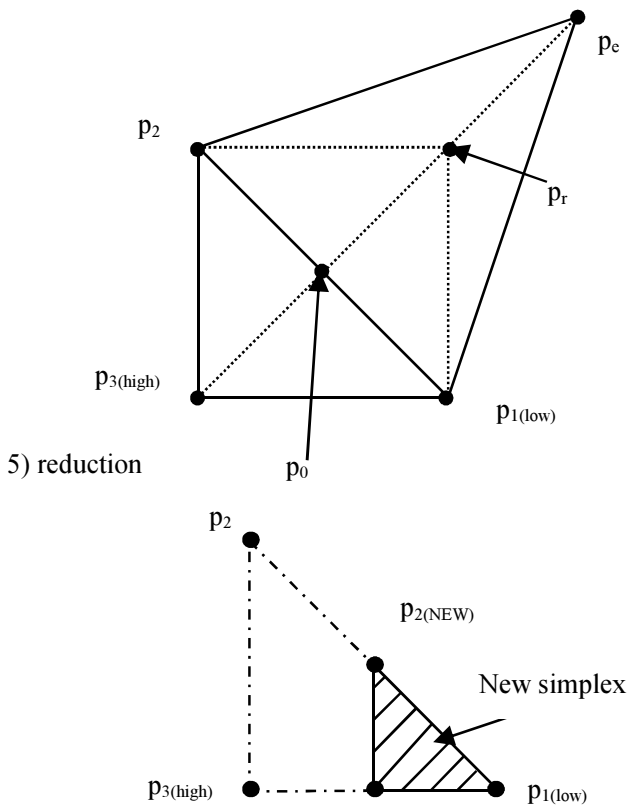

2) reflection

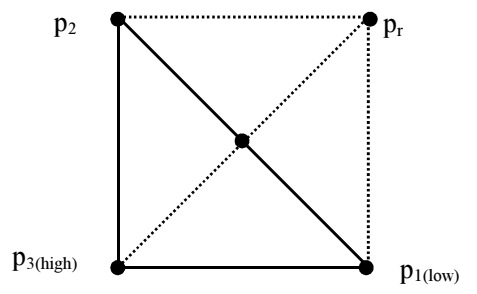

4) contraction

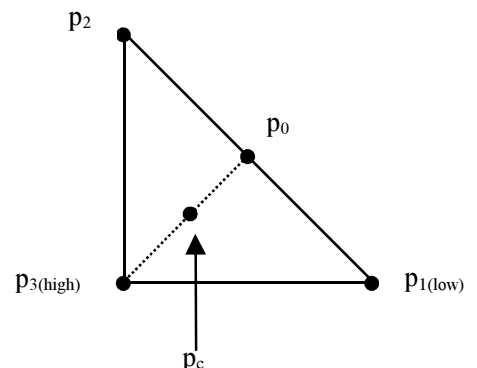

$\mathrm{p}_{3 \text { (NEW) }}$

Fig. 1. The proceeding in one iteration

\section{Methodology}

The task is to fit circular arc into a set of measured points. Figure 2 depicts the geometrical relationship of arc fitting. Vertices $\mathrm{A}$ and $\mathrm{B}$ are the point of arc tangency, vertex $\mathrm{W}$ is a point of intersection of the tangents. These vertices form a 
sections AW and BW. From coordinates of these vertices was calculated switch angle $(\gamma)$. The measured points on the arc were marked by a squares with "*" in inside, while measured points on the straight sections were marked by empty squares.

One of the problems encountered when developing the task is to select among all measured points the group of points placed on the arc. In order to solve this problem the transformation of the coordinate system was carried out. The new coordinate system has a beginning at the point $\mathrm{W}, \mathrm{X}$ axis is a bisector $\mathrm{WO}$ and the $\mathrm{Y}$ axisis orthogonal to the $\mathrm{X}$. The location of the new coordinate system depicts Figure 2.

It is assumed that $\mathrm{X}_{\min }$ is the distance $\mathrm{s}$ between $\mathrm{W}$ and $\mathrm{S}$ (point placed on arc with equal distance from the beginning and end of arc). Then the initial values of parameters and chord of arc are computed by the formulas 6-9:

$$
\begin{gathered}
R=\frac{s}{\left(\cos \frac{\gamma}{2}-1\right)} \\
x_{0}=s\left(\begin{array}{c}
\left.1+\frac{1}{\left(\cos \frac{\gamma}{2}-1\right)}\right) \\
y_{0}=0 \\
a=s \frac{\sin \frac{\gamma}{2}}{\left(\cos \frac{\gamma}{2}-1\right)}
\end{array}\right.
\end{gathered}
$$

where:

$x_{0}, y_{0}-x$ and $y$ coordinates of the center of the circular arc,

$R$ - radius of circular arc,

$a-$ half of chord of circular arc.

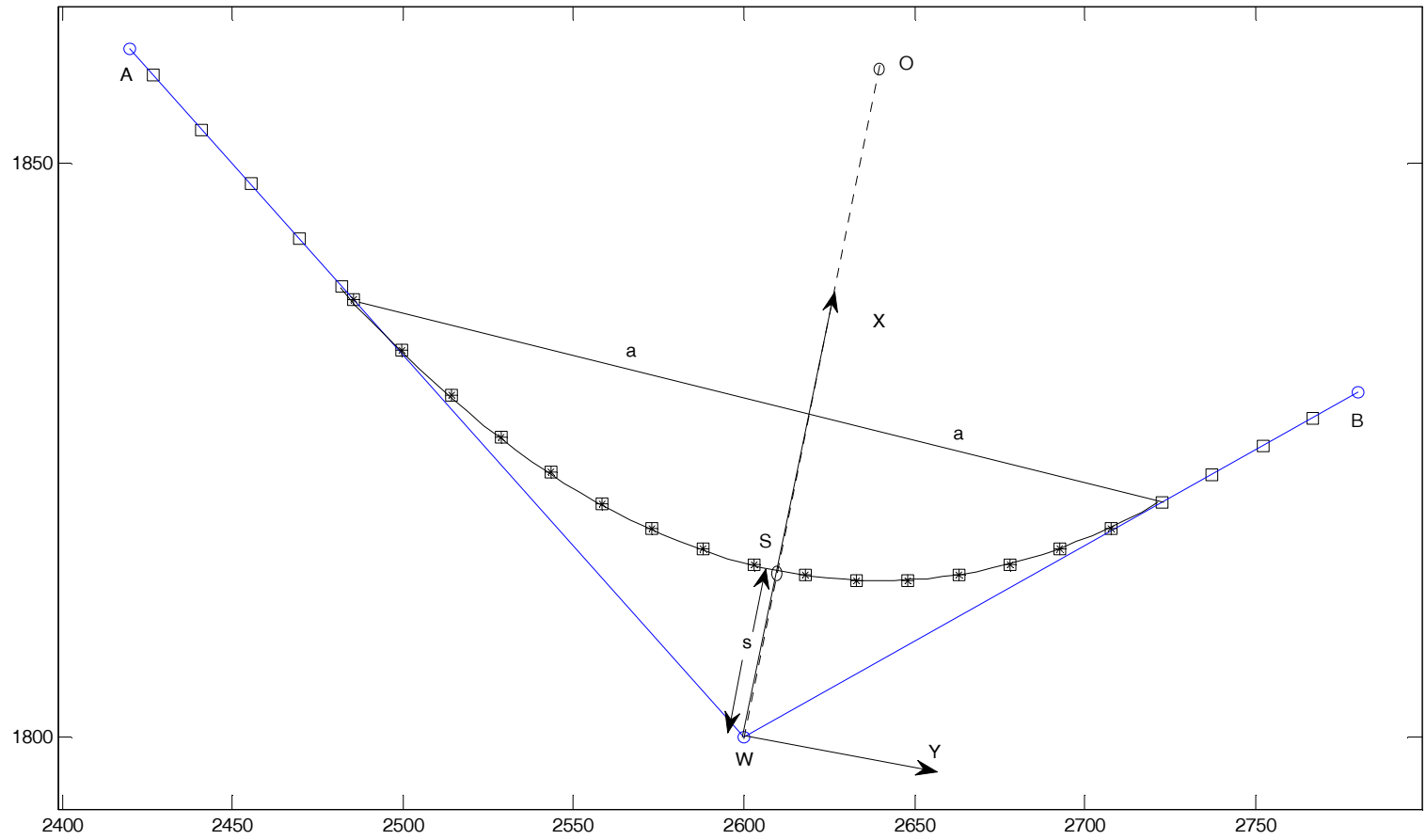

Fig. 2. Local coordinate system

The beginning and the end of the arc can be easily pointed in such a coordinate system. First and last point of the arc are determined by the length of parameter a (points with the values of $Y$ coordinates from $-\mathrm{a}$ to a). After determining the group of points for fitting, the reverse transformation into initial coordinate system can be performed.

The observation equations can be presented as follows:

$$
\left(x_{i}-x_{0}\right)^{2}+\left(y_{i}-y_{0}\right)^{2}=R^{2},
$$


where:

$x_{i}, y_{i}-x$ and $y$ coordinates of the i-th measured point.

Apart from the observation equations, the conditional equations are used.

$$
\begin{gathered}
\left(x_{w}-x_{0}\right)^{2}+\left(y_{w}-y_{0}\right)^{2}=R^{2}+R^{2} \tan ^{2} \frac{\gamma}{2} \\
\arctan \frac{\left(y_{w}-y_{0}\right)}{\left(x_{w}-x_{0}\right)}=\arctan \frac{\left(y_{w}-y_{P}\right)}{\left(x_{w}-x_{P}\right)}-\frac{1}{2}(\pi-\gamma)
\end{gathered}
$$

where:

$x_{w} y_{w}-x$ and $y$ coordinates of the $\mathrm{W}$ vertex.

Eqn (11) ensures that circular arc is tangent to the axis of the way (AW and BW sections), before and after curvature. Formula 12 ensures that the location of the center of the arc is on the bisector angle at vertex (AW and BW sections). The fulfillment of conditional equations is ensured by the use of method of large weights.

In the next step the objective function is formed:

$$
\psi=\sum_{i+1}^{n}\left(V_{i}^{2}+p V_{\Delta 1}^{2}+p V_{\Delta 2}^{2}\right)
$$

where:

$$
\begin{gathered}
V_{i}=\sqrt{\left(x_{i}-x_{0}\right)^{2}+\left(y_{i}-y_{0}\right)^{2}}-R \\
V_{\Delta 1}=\sqrt{\left(x_{w}-x_{0}\right)^{2}+\left(y_{w}-y_{0}\right)^{2}} \times \cos \frac{\gamma}{2}-R \\
V_{\Delta 2}=\arctan \frac{\left(y_{w}-y_{p}\right)}{\left(x_{w}-w_{p}\right)}-\arctan \frac{\left(y_{w}-y_{0}\right)}{\left(x_{w}-x_{0}\right)}+\frac{(\pi-\gamma)}{2}
\end{gathered}
$$

and $p=1000$ is a large weight to ensure the fulfillment of condition equations.

Alternatively the objective function can be presented in the matrix notation:

$$
\Psi=\mathrm{V}^{\mathrm{T}} \mathrm{PV}=\min ,
$$

where:

$$
V=\left[\begin{array}{c}
V_{i} \\
n \times 1 \\
V_{\Delta 1} \\
V_{\Delta 2}
\end{array}\right]
$$

and matrix P is:

$$
\mathrm{P}=\left[\begin{array}{cc}
\underset{\mathrm{n} \times \mathrm{n}}{\mathrm{N}} & 0 \\
0 & 1000
\end{array}\right]
$$

The value of objective function is calculated according to the formula (13) or (17) in Nelder-Mead simplex algorithm.

Through the optimal choice of control parameters such as the size of the first simplex and terminate criterion of iterative process a problem of ensure the convergence and the efficiency of optimization task was done. Implementation of this algorithm was made in Matlab ${ }^{\circledR}$. In next section the results of test are presented.

\section{Experiment}

The initial values of parameters calculated from Eqn (10) are listed in Table 1: 
Table 1. Approximate parameters of arc

\begin{tabular}{ll}
\hline Parameter & Value $(\mathrm{m})$ \\
\hline$R$ & 498.832 \\
$x_{0}$ & 2312.445 \\
$y_{0}$ & 2639.288 \\
\hline
\end{tabular}

The initial simplex was formed as a set of 4 points:

- $\left(\mathrm{R}, \mathrm{x}_{0}, \mathrm{y} 0\right)$,

- $\left(\mathrm{R}+10, \mathrm{x}_{0}, \mathrm{y}_{0}\right)$,

- $\left(\mathrm{R}, \mathrm{x}_{0}+10, \mathrm{y}_{0}\right)$,

- $\left(\mathrm{R}, \mathrm{x}_{0}, \mathrm{y}_{0}+10\right)$.

It was assumed that the iterative, computational process should terminate when one of the three criterions is satisfied:

- $\quad$ tolf $=0.0001$

- tolx $^{=0.0001}$

- $\operatorname{maxiter}=600$

where:

- $\quad$ tolf $=\Psi \max -\Psi \min \quad$ (difference between maximal and minimal value of objective function in the points of simplex),

- $\operatorname{tolx}=\mathrm{Xmax}-\mathrm{Xmin}$ (difference between maximal and minimal value of equivalent parameter in the points of simplex),

- maxiter - maximal number of iterations.

In the experiment the second criterion (tolx $=0.0001$ ) was achieved.

The final values of parameters were obtained after 181 iterations. They are listed in Table 2. The differences between approximate and final values of parameters amount to about eighty - one hundred centimeters.

Table 2. Comparison of parameters task

\begin{tabular}{llll}
\hline Parameter & Determined value $(m)$ & Approximated value $(m)$ & Difference $(m)$ \\
\hline $\mathrm{R}$ & 499.645 & 498.832 & 0.813 \\
$\mathrm{x}_{0}$ & 2313.254 & 2312.445 & 0.809 \\
$\mathrm{y}_{0}$ & 2640.274 & 2639.288 & 0.986 \\
\hline
\end{tabular}

The table below contains the residuals calculated on the basis of final parameters from formulas (14-16):

Table 3. Residuals for measuring points

\begin{tabular}{|c|c|c|c|c|c|c|c|c|c|c|c|c|c|c|c|c|}
\hline No & 1 & 2 & 3 & 4 & 5 & 6 & 7 & 8 & 9 & 10 & 11 & 12 & 13 & 14 & 15 & 16 \\
\hline 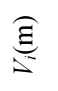 & $\begin{array}{l}\mathscr{8} \\
\stackrel{0}{0} \\
\dot{p}\end{array}$ & $\begin{array}{l}\qquad \\
ْ \\
\circ \\
0\end{array}$ & $\begin{array}{l}\hat{8} \\
\stackrel{0}{0}\end{array}$ & $\begin{array}{l}\overline{0} \\
\stackrel{0}{0}\end{array}$ & $\stackrel{0}{\circ}$ & $\begin{array}{l}\hat{8} \\
\stackrel{0}{0} \\
\end{array}$ & 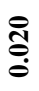 & $\begin{array}{l}\text { } \\
\stackrel{8}{0} \\
\dot{\varphi}\end{array}$ & 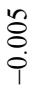 & $\begin{array}{l}\text { ¿̊ } \\
\stackrel{0}{0}\end{array}$ & $\begin{array}{l}\mathscr{8} \\
0 \\
0 \\
0\end{array}$ & $\begin{array}{l}\text { ¿े } \\
\stackrel{0}{0}\end{array}$ & ڤ્o & \begin{tabular}{l}
$n$ \\
\hdashline \\
0 \\
0
\end{tabular} & 용 & $\begin{array}{l}n \\
0 \\
0\end{array}$ \\
\hline
\end{tabular}

The resulting sum of the squared residuals $\left(V^{T} P V\right)$ amounts to 0.0013 . The maximum residual $\left(V_{\max }\right)$ is not more than twenty millimeters, while the minimum residual $\left(V_{\min }\right)$ is 0.0001 . 


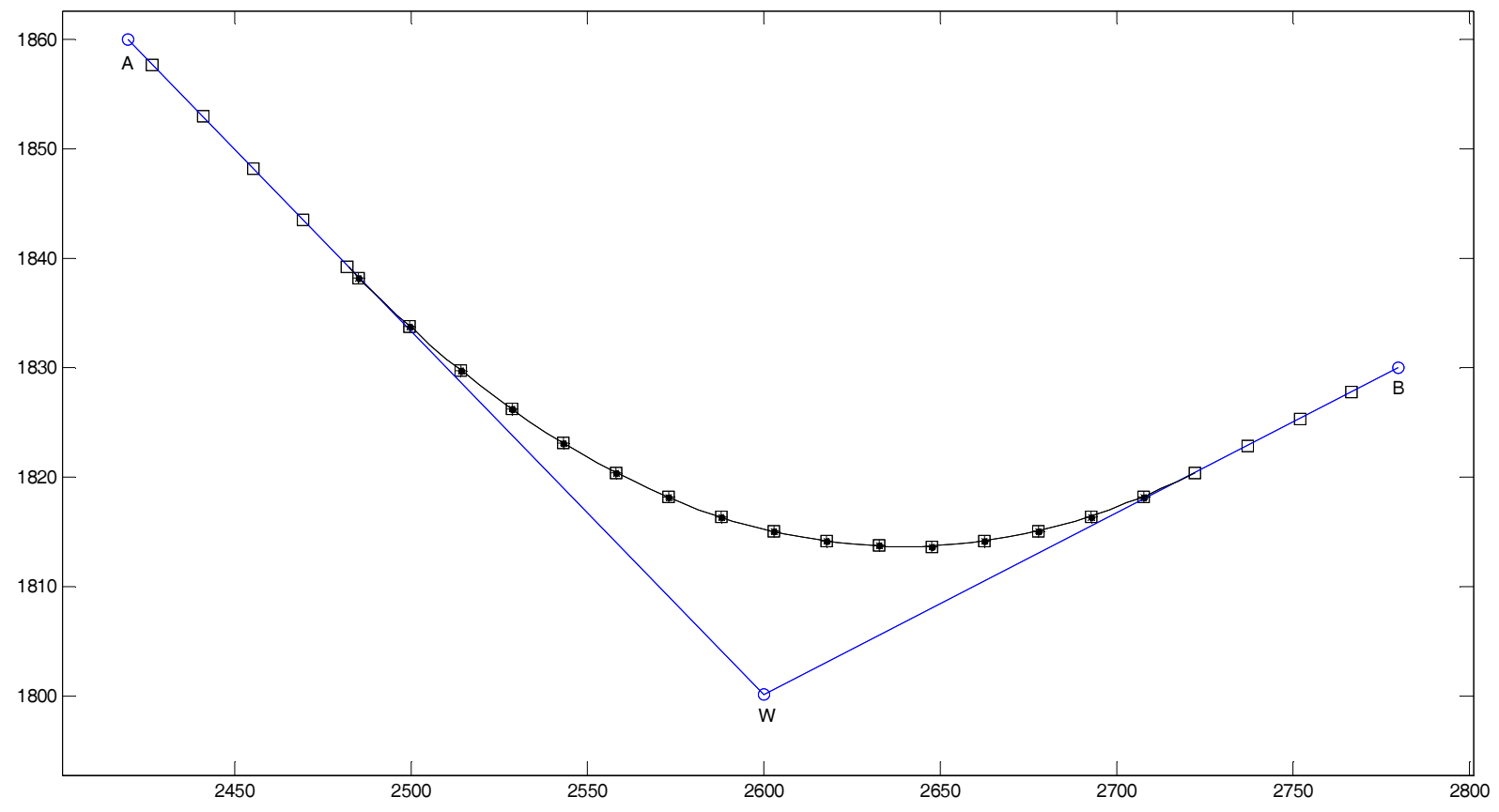

Fig. 3. Fitting of circular arc into a set of points

The graphical interpretation of the results is presented in Figure 3. The measured points on the arc were marked by a squares with "*" in inside, while measured points on the straight sections were marked by empty squares. The circular arc was marked by a line.

\section{Summary}

The article presents a new approach to fitting railway track into a set of measured points. The new method uses the NelderMead simplex method for minimization of the residuals of fitting points. The successive stages of implementation of the new method were described. This method was tested on a set of points. The resultsof the example were presented and analysed. The proposed approach is computationally efficient and stable in the meaning of obtaining one unique and optimal solution.

\section{References}

[1] Koc, W.; Specht, C.; Chrostowski, P.; Palikowska, K. 2012. The Accuracy Assessment of Determining the Axis of Railway Track Basing on the Satellite Surveying, Archives of Transpor 24(3): 307-320.

[2] Kozieł, S.; Yang, X. S. 2011. Computational, optimization, methods and algorithms, Studies is coputational inteligence vol. 356 : 5.

[3] Lenda, G.; Strach, M. 2012. Determining the geometry of rail routes using the approximating spline functions, $P A K$ (12): $1082-1086$.

[4] Nelder, A.; Mead, R. 1965. A simplex method for function minimization, Computer Journal 7: 308-313. http://dx.doi.org/10.1093/comjnl/7.4.308

[5] Bałuch, H. 1978. Diagnosis surface rail. Warsaw: Publisher Transport and Communications.

[6] Kobryń, A. 2009. Polynomial shaping the transition curves. Bialystok: Department of Publications and Printing Bialystok University of Technology.

[7] Collective work edited by Laudyn, I. 1993. Engineering geodesy, Vol. III, Warsaw: Polish Enterprise Cartography Publishing them E. Romera, 445497.

[8] Kędra, Z. 2005. Methods of axis rails realignment, Gdansk. Speech at the jubilee scientific session Railway Engineering Department, 171-181. 